\title{
LEDs Electrifying Horticultural Science: Proceedings from the 2014 Colloquium and Workshop
}

\author{
Gioia D. Massa ${ }^{1}$ \\ NASA, Mail Code: UB-A, Kennedy Space Center, FL 32899 \\ Jeff Norrie \\ Acadian Seaplants Limited, 30 Brown Avenue, Dartmouth, Nova Scotia, Canada, B3B 1X8
}

Additional index words. commercial technology, controlled environment, light emitting diode, light quality

The use of Light Emitting Diode (LED) technology for research and commercial horticulture has undergone exponential growth in the past decade. In 2007, the American Society for Horticultural Science (ASHS) held a workshop on "LED Lighting for Horticulture" that generated significant interest. It was, by all accounts, an excellent session, with the subsequent proceedings an oft-cited series of review articles shedding much needed attention on a rapidly developing application in horticulture (Bourget, 2008; Folta and Childers, 2008; Massa et al., 2008; Morrow, 2008; Wheeler, 2008).

From that springboard, horticultural applications for LED technology have advanced in recent years at, dare we say it, the speed of light. With developments in spectral quality, intensity, architecture, customizable light "recipes," species-specific lighting protocols, and a myriad of plant-specific interactions, opportunities to commercialize these technologies have never been more focused. Add to that improvements in lighting efficiencies and affordability, and LED technologies are increasingly becoming mainstream. Thus, the electric-lighting horticultural community decided that it was time for an update on new research and applications of this versatile and useful technology.

The 2014 ASHS meeting in Orlando provided a fantastic opportunity to bring together a number of critical thinker-researchers to discuss the current state-of-the-art. A colloquium entitled "The Importance of Light Quality for High Value Plant Products" was held in conjunction with a workshop, "Advances in Commercial-ready LED Technologies for Horticulture." Other highlights included an industry/research "Roundtable

\footnotetext{
Received for publication 26 Jan. 2015. Accepted for publication 6 Mar. 2015.

We appreciate the support of ASHS and all the speakers, authors, and audiences from both sessions. The colloquium was jointly sponsored by the Growth Chambers and Controlled Environments (CEWG), Floriculture (FLOR), Quality, Safety, and Health Properties (QUAL), and Vegetable Crops Management (VCM) working groups. Many thanks to the colloquium organizing committee members Marc van Iersel, Dean Kopsell, Stephanie Burnett, and Joey Norikane.

${ }^{1}$ Corresponding author. E-mail: gioia.massa@nasa. gov.
}

Discussion on the Standardization of LED Lighting for Horticultural Applications," numerous poster and oral presentations on related topics, and of course an active trade show featuring several lighting manufacturers and end-users.

LEDs are just the latest useful tool in the arsenal of horticultural researchers, and we stand on a solid foundation built by researchers who have come before us. Manipulating light quality as a mechanism for adjusting the growth of plants goes back to the earliest days of plant physiology, when prisms were used to separate the visible spectrum and researchers identified colors of light important in plant responses. The earliest such inquiry by Sebastiano Poggioli, published in 1817, studied effects of red and violet light on leaf growth and position in Mimosa pudica and Raphanus rusticanus. Many subsequent examinations of plant responses to light quality were performed in the second half of the 19th-century, including those by some of the preeminent founders of modern plant physiological research, including Julius von Sachs (1864) and Charles and Francis Darwin (1880).

During the same period, the use of electric lamps for plant growth began in the $1860 \mathrm{~s}$ and expanded as lighting technologies developed. Wheeler (2008) provides a brief history of the development and adoption of these technologies for plant research. Shortly after electric lamps were developed, they were first used in greenhouse experimentation by Siemens (1880) in England, and in the United States, by the first president of the ASHS, L.H. Bailey (1890). From there, as with other innovations, progressive horticulturists adopted, adapted, experimented, and optimized to bring us to our present state.

The horticultural community has closely followed the development of lighting technologies for other larger markets, such as household and industrial lighting, automotive and traffic lights. Many of the lights used in commercial agriculture and agricultural research were never designed for plants, but we have found uses perhaps never dreamed of by their inventors.

The 2014 colloquium on the importance of light quality for high value plant products focused on active areas of research on light impacts in specialty crop production systems including vegetables, floriculture and other ornamentals, and bio-pharmaceuticals. Fundamental research focusing on using LED technologies to understand the mechanisms behind basic plant responses to light helps untangle the respective roles of the photoreceptor and photosynthetic signaling networks. The knowledge gained can then be harnessed for plant production applications to benefit humanity. Researchers are looking at modifying plant yield, size, shape, color, flower number, flavor, nutritional characteristics, and even pharmaceutical "active ingredient" levels using light. Season extension and year-round factory farming approaches are now more feasible based on the capability to select energy efficient lighting systems, customized crops, and other parameters of interest.

Moreover, we are able to follow this work through to include a focus on commercially ready_or almost ready_LED technologies, with perspectives from several leading "industry" scientists or those working closely on industry projects. Exploring these advances in research provides insight into those technologies that are expected to see commercial success in the short to medium term, and provide economic benefit to all horticultural stakeholders. Given that we all enjoy nutritious, fresh fruits and vegetables and high quality ornamentals at a reasonable price, the benefits to our stakeholders at large are obvious.

We would like to thank the ASHS for the opportunity to bring together such an excellent cast of researchers and scientists, and to dovetail the colloquium on light quality research focused on horticultural applications. For these proceedings, we bring together some of the perspectives and practices of individuals who are advancing this frontier for horticultural sciences. Kevin Folta and Sofia Carvalho present "Photoreceptors and control of horticultural plant traits," discussing the fundamental mechanisms of how various wavelengths activate signal transduction mechanisms to control plant growth, development, physiology, and metabolite accumulation. Tessa Pocock presents "Light emitting diodes and the modulation of specialty crops: Light sensing and signaling networks in plants." She describes the components of the different photoreceptor and photosynthetic sensing and signaling networks, and discusses how 
understanding and using those can guide the selection and use of LEDs for plant growth. Focusing on applications, Dean Kopsell, Carl Sams, and Robert Morrow present "Blue wavelengths from LED lighting increase nutritionally important metabolites in specialty crops." They discuss numerous studies using blue light to modulate nutritionally important pigments, glucosinolates, and mineral elements in fresh produce. Joey Norikane discusses "The potential of LEDs in plant-based bio-pharmaceutical production." He describes the different phases of bio-pharmaceutical crop production and he discusses how LED technology can be used to optimize the cropping environment at different stages of growth and development. Cary Mitchell discusses his "Academic research perspective of LEDs for the horticulture industry." He presents a general overview of interest in LEDs for horticulture, talks about the role of research, and discusses the evolution of LED arrays for different crop scenarios. Gary Stutte presents "Commercial transition to LEDs: a pathway to higher value products" in which he describes his perspective on what is happening with LEDs, how research and technology development have grown dramatically, and what factors influenced them. He discusses policy and economic aspects driving LED innovation, and reflects on how we can use this dynamic area to benefit horticulture.

Hopefully, these proceedings, coupled with the recorded ASHS presentations from the colloquium and workshop, will act as another catalyst to advance our understanding and application of LED technologies in horticulture and to inspire the next generation of researchers to discover, innovate, and adapt tools to further advance this exciting frontier.

\section{Literature Cited}

Bailey, L.H. 1890. Influence of electric light on greenhouse plants. Cornell University Agriculture Experiment Station Bulletin 30.

Bourget, C.M. 2008. An Introduction to Lightemitting Diodes. HortScience 43:19441946.
Darwin, C. and F. Darwin. 1880. The Power of movement in plants. Appleton and Company, New York, NY.

Folta, K.M. and K.S. Childers. 2008. Light as a growth regulator: Controlling plant biology with narrow-bandwidth solid-state lighting systems. HortScience 43:1957-1964.

Massa, G.D., H.-H. Kim, R.M. Wheeler, and C.A. Mitchell. 2008. Plant productivity in response to LED lighting. HortScience 43: 1951-1956.

Morrow, R.C. 2008. LED lighting in horticulture. HortScience 43:1947-1950.

Poggioli, S. 1817. Della influenza che ha il raggio magnetico sulla vegetatione delle piante. Opuscoli Scientifici (Bologna) 1:9-23.

Sachs, J. 1864. Wirkungen farbigen lichts auf pflanzen. Bot. Zeitung 47:353-358.

Siemens, C.W. 1880. On the influence of electric light upon vegetation and on certain physical principles involved. Proc. R. Soc. Lond. 30:210-219.

Wheeler, R.M. 2008. A historical background of plant lighting: An introduction to the workshop. HortScience 43:1942-1943. 